\title{
Solid wall effect on the transport coefficients of gases
}

\author{
FEI Fei, FAN Jing ${ }^{*} \&$ JIANG JianZheng \\ State Key Laboratory of High Temperature Gas Dynamics, Institute of Mechanics, Chinese Academy of Sciences, Beijing 100190, China
}

Received January 13, 2012; accepted February 29, 2012; published online April 20, 2012

\begin{abstract}
The diffusion, viscosity and thermal conductivity coefficients of gases between two parallel solid walls have been obtained analytically based on the Green-Kubo relation under a hard-sphere model. They decrease nonlinearly as the Knudsen number defined as the ratio of the mean free path to the wall distance increases. This theoretical prediction was in good agreement by the DSMC results.
\end{abstract}

transport coefficient, solid wall effect, Green-Kubo relation, Chapman-Enskog theory, DSMC

PACS number(s): 47.45.Ab, 05.20.Dd, 05.10.Ln

Citation: Fei F, Fan J, Jiang J Z. Solid wall effect on the transport coefficients of gases. Sci China-Phys Mech Astron, 2012, 55: 927-932, doi: $10.1007 / \mathrm{s} 11433-012-4733-6$

Transport phenomenon is an essential feature of fluids [1]. In classical fluid dynamics, the fluxes of mass, momentum, and energy are assumed to be proportional to the gradients of the concentration, velocity and temperature, respectively, i.e.,

$$
j=D \frac{\mathrm{d} n}{\mathrm{~d} y}, \quad \tau=\eta \frac{\mathrm{d} u}{\mathrm{~d} y}, \quad q=\kappa \frac{\mathrm{d} T}{\mathrm{~d} y},
$$

where $n, u$ and $T$ are the concentration, velocity and temperature, and $D, \eta$ and $\kappa$ are the coefficients of diffusion, viscosity and thermal conductivity, respectively, which are phenomenological and usually need to be determined through experimental data.

The Chapman-Enskog (C-E) theory [2] was one of the most important achievements of kinetic studies of gases. It established the relationship between the macroscopic transport coefficients and molecular motion, which has been validated and verified by a number of measured data $[1,2]$. For hard sphere molecules, according to the C-E theory, the transport coefficients can be written as:

*Corresponding author (email: jfan@imech.ac.cn)

$$
\eta=\frac{5}{16} \frac{\sqrt{\pi m k_{\mathrm{B}} T}}{\sigma_{T}}, \frac{\kappa}{\eta}=\frac{5 R}{2(\gamma-1)}, \quad \frac{D}{\eta}=\frac{6}{5 \rho},
$$

where $m$ and $\sigma_{T}$ are the molecular mass and collision cross section, respectively, $k_{\mathrm{B}}$ is the Boltzmann constant, $R=k_{\mathrm{B}} / m$, and $\gamma$ is the specific heat ratio.

Another approach to determine transport coefficients originated from Einstein [3]. In the study of the Brownian motion, he demonstrated that the mean square displacement of dust is proportional to the diffusion coefficient of the ambient fluid as time is large enough, that is $\left\langle\Delta r^{2}\right\rangle=6 D t$ $(t \rightarrow \infty)$. The Einstein relation reveals the relevance between the dissipation and fluctuation of fluids. Later in the 1950s, Kubo et al. [4] developed a linear response theory that extended the Einstein relation. Based on the linear response theory, the transport coefficients have the following expressions that apply to both liquids and gases [4-6]:

$$
\begin{gathered}
D=\int_{0}^{\infty}\langle v(0) v(t)\rangle \mathrm{d} t, \\
\eta=\frac{1}{k_{\mathrm{B}} V T} \int_{0}^{\infty}\left\langle J_{x y}^{\eta}(t) J_{x y}^{\eta}(0)\right\rangle \mathrm{d} t,
\end{gathered}
$$




$$
\kappa=\frac{1}{k_{\mathrm{B}} V T^{2}} \int_{0}^{\infty}\left\langle J_{y}^{\kappa}(0) J_{y}^{\kappa}(t)\right\rangle \mathrm{d} t,
$$

where $V$ and $N$ are the volume and molecular number of a system, respectively, \langle\rangle denotes an ensemble average, $J_{x y}^{\eta}(t)=\sum_{i=1}^{N} m v_{i x} v_{i y}$ is the microscopic expression of shear stress flux, and $J_{y}^{\kappa}(t)=\sum_{i=1}^{N}\left[\frac{1}{2} m v_{i}^{2}-\frac{5}{2} k T\right] v_{i y}(t)$ is the microscopic expression of energy flux.

The transport coefficients of gases can be calculated based on the Green-Kubo (G-K) relation (eqs. (3a)-(3c)) using the direct simulation Monte Carlo (DSMC) method [7]. In the DSMC method, gases are represented by a number of model molecules whose trajectories are tracked by a computer. The reliability and accuracy of DSMC were well tested and verified theoretically and experimentally during the past 50 years. An example is shown in Table 1: the transport coefficients of argon gas at the standard conditions obtained by our DSMC calculations are compared to the classical C-E theory (2). The computational domain of DSMC is a square, and the boundary conditions are all periodic. The side length of $10 \lambda$ is uniformly divided into 50 cells, and the time step $\Delta t=0.1 \tau_{\mathrm{c}}$, where $\lambda$ and $\tau_{\mathrm{c}}$ are the mean free path and mean collision time of argon molecules, respectively. Initially, 50 simulated molecules in an equilibrium state at rest are assigned into each cell following the standard procedures of DSMC. The molecular mass and specific heat ratio of argon are $6.63 \times 10^{-26} \mathrm{~kg}$ and $5 / 3$, respectively [2,7]; for the hard-sphere model, its collision cross section is $4.2 \times 10^{-19} \mathrm{~m}^{2}$ [2,7]. Table 1 demonstrates that the DSMC results agree well with the C-E theory, which also verifies our program.

Gas flows in engineering context often involve various interactions between gases and solid walls. They are one of the most important sources of many flow phenomena and patterns. It is easily understood that a solid wall will affect the transport coefficients of gases adjacent to it, because the gas-solid interaction is far different from that between gaseous molecules themselves [8,9]. It is a focus of the present paper to make a quantitative analysis to view such an effect.

Before a further analysis, we firstly perform a preliminary calculation to look at how much solid walls affect the transport coefficients of their neighboring gases. The calculation is similar to Table 1, and the main difference is the upper and lower sides that are replaced by solid walls. In

Table 1 Comparison of diffusion, viscosity and thermal conductivity coefficients of argon gas in the standard conditions obtained by the DSMC calculations based on the G-K relation (3) and the C-E theory (2)

\begin{tabular}{ccc}
\hline Coefficients & DSMC + G-K relation & C-E theory \\
\hline$D\left(\mathrm{~cm}^{2} / \mathrm{s}\right)$ & $1.43 \times 10^{-1}$ & $1.43 \times 10^{-1}$ \\
$\mu\left(\mathrm{Ns} / \mathrm{m}^{2}\right)$ & $2.15 \times 10^{-5}$ & $2.12 \times 10^{-5}$ \\
$\kappa(\mathrm{J} / \mathrm{msK})$ & $1.69 \times 10^{-2}$ & $1.65 \times 10^{-2}$ \\
\hline
\end{tabular}

kinetic studies [1-7], a solid wall is often assumed to be fully diffusely reflecting, as does it in this paper. The wall temperatures are $273 \mathrm{~K}$, which is the same as the argon gas. As an example, the procedure to calculate the viscosity coefficient is described as follows:

(1) At the $i$ th time step $t_{i}, t_{i}=i \Delta t\left(i=1,2, \ldots N_{0}\right)$, the microscopic shear stress $J_{x y}^{\eta}\left(t_{i}\right)=\sum_{i=1}^{N} m v_{i x} v_{i y}$ is obtained through a statistical mean of the known velocity components of each simulated molecule;

(2) For certain time interval $\Delta t^{*}$, the autocorrelation function of viscosity at time $t_{i}$ is obtained by an ensemble averaging, i.e.,

$$
\left\langle J_{x y}^{\eta}\left(t_{i}\right) J_{x y}^{\eta}(0)\right\rangle=\frac{1}{N_{t_{i}}+1} \sum_{k=0}^{N_{t_{i}}} J_{x y}^{\eta}\left(t_{i}+k \Delta t^{*}\right) J_{x y}^{\eta}\left(k \Delta t^{*}\right),
$$

where $\quad N_{t_{i}}=\left(N_{0} \Delta t-t_{i}\right) / \Delta t^{*}$, and $\Delta t^{*}=100 \Delta t$ in the present calculations.

(3) Substitute $\left\langle J_{x y}^{\eta}\left(t_{i}\right) J_{x y}^{\eta}(0)\right\rangle$ into the G-K relation (3b), and numerically integrate to obtain the viscosity coefficient.

As shown in Table 2, the transport coefficients of argon gas decrease significantly as the distance between the upper and lower plates $L$ decreases. Compared to the viscosity coefficient without the effect of solid walls $2.15 \times 10^{-5} \mathrm{Ns} / \mathrm{m}^{2}$ given in Table $1, \mu^{*}$ ( the superscript $*$ denotes the effect of solid walls) decreases about $2 \%, 19 \%$ and $69 \%$ when $L=$ $100 \lambda, 10 \lambda$ and $\lambda$, respectively.

\section{Transport coefficients of gases between two parallel solid walls}

The velocity autocorrelation function of gaseous molecules in a system,

$$
C^{D}(t) \equiv\left\langle v_{x}(0) v_{x}(t)\right\rangle=\frac{1}{N} \sum_{i=1}^{N} v_{x i}(0) v_{x i}(t)
$$

with the initial value

$$
C^{D}(0)=\left\langle v_{x}^{2}\right\rangle=k_{\mathrm{B}} T / m=0.5 c_{m}^{2},
$$

where $c_{m}=\sqrt{2 k_{\mathrm{B}} T / m}$ is the most probable speed of thermal motion.

During a time interval $\Delta t$, the velocity autocorrelation

Table 2 Transport coefficients of argon in the standard condition between two solid plates at three different distances obtained by the DSMC calculations based on the G-K relation (eqs. (3a)-(3c))

\begin{tabular}{cccc}
\hline Coefficients & $100 \lambda$ & $10 \lambda$ & $\lambda$ \\
\hline$D^{*}\left(\mathrm{~cm}^{2} / \mathrm{s}\right)$ & $1.41 \times 10^{-1}$ & $1.31 \times 10^{-1}$ & $7.65 \times 10^{-2}$ \\
$\mu^{*}\left(\mathrm{Ns} / \mathrm{m}^{2}\right)$ & $2.10 \times 10^{-5}$ & $1.74 \times 10^{-5}$ & $6.70 \times 10^{-6}$ \\
$\kappa^{*}(\mathrm{~J} / \mathrm{msk})$ & $1.60 \times 10^{-2}$ & $1.17 \times 10^{-2}$ & $3.25 \times 10^{-3}$ \\
\hline
\end{tabular}


function near a solid wall changes owing to two different mechanisms. One is collisions between the gaseous molecules themselves, and the other is the interaction between the gaseous molecules and walls. Therefore, we have

$$
\Delta C^{D}\left(t_{0}\right)=C^{D}\left(t_{0}+\Delta t\right)-C^{D}\left(t_{0}\right)=\Delta C_{\text {bulk }}^{D}\left(t_{0}\right)+\Delta C_{\text {wall }}^{D}\left(t_{0}\right)
$$

For the first mechanism, the velocity autocorrelation function decays exponentially due to the collisions between the gaseous molecules themselves [6],

$$
\begin{aligned}
\Delta C_{\text {bulk }}^{D}\left(t_{0}\right) & =\left[C^{D}\left(t_{0}+\Delta t\right)-C^{D}\left(t_{0}\right)\right]_{\text {bulk }} \\
& =C^{D}\left(t_{0}\right) \mathrm{e}^{-\vartheta \Delta t}-C^{D}\left(t_{0}\right)=-C^{D}\left(t_{0}\right) \vartheta \Delta t,
\end{aligned}
$$

where $\vartheta=C^{D}(0) / D$.

For the second mechanism, during the interval $\Delta t$, all the gaseous molecules in the system can be divided into two parts: one collide with the walls, while the other do not. Consequently,

$$
\begin{aligned}
& C^{D}\left(t_{0}\right)= \frac{1}{N} \sum_{i=1}^{N} v_{x i}(0) v_{x i}\left(t_{0}\right) \\
&=\frac{1}{N}\left(\sum_{i=1}^{N_{\text {wall }}} v_{x i}(0) v_{x i}\left(t_{0}\right)+\sum_{i=N_{\text {wall }}+1}^{N} v_{x i}(0) v_{x i}\left(t_{0}\right)\right) \\
& C^{D}\left(t_{0}+\Delta t\right)= \frac{1}{N} \sum_{i=1}^{N} v_{x i}(0) v_{x i}\left(t_{0}+\Delta t\right) \\
&= \frac{1}{N}\left(\sum_{i=1}^{N_{\text {wall }}} v_{x i}(0) v_{x i}\left(t_{0}+\Delta t\right)\right. \\
&\left.+\sum_{i=N_{\text {wall }}+1}^{N} v_{x i}(0) v_{x i}\left(t_{0}+\Delta t\right)\right)
\end{aligned}
$$

where $N_{\text {wall }}$ is the number of molecules striking the solid walls during the time interval, which can be expressed as follows $[1,2,7]$ :

$$
N_{\text {wall }}=2 \times \frac{1}{4} n \bar{c} \Delta t=\frac{1}{2} \times \frac{N}{L} \times \frac{2 c_{m}}{\sqrt{\pi}} \Delta t=N \times \frac{c_{m} \Delta t}{\sqrt{\pi} L} .
$$

When we are concerned only the effect of the solid walls, the velocity autocorrelation function of the part of the molecules without colliding with the walls will remain unchanged. Thus,

$$
\begin{aligned}
\Delta C_{\text {wall }}^{D}\left(t_{0}\right) & =\left[C^{D}\left(t_{0}+\Delta t\right)-C^{D}\left(t_{0}\right)\right]_{\text {wall }} \\
& =\frac{1}{N} \sum_{i=1}^{N_{\text {wall }}} v_{x i}(0) v_{x i}\left(t_{0}+\Delta t\right)-\frac{1}{N} \sum_{i=1}^{N_{\text {wall }}} v_{x i}(0) v_{x i}\left(t_{0}\right) .
\end{aligned}
$$

According to the diffuse reflection model, the velocities of a molecule before and after hitting a wall are irrelevant, i.e.,

$$
\sum_{i=1}^{N_{\text {wall }}} v_{x i}(0) v_{x i}\left(t_{0}+\Delta t\right)=0
$$

Substitution of eq. (11) into eq. (10) yields

$$
\begin{aligned}
\Delta C_{\text {wall }}^{D}\left(t_{0}\right) & =-\frac{1}{N} \sum_{i=1}^{N_{\text {wall }}} v_{x i}(0) v_{x i}\left(t_{0}\right) \\
& =-\frac{N_{\text {wall }}}{N} \times \frac{1}{N_{\text {wall }}} \sum_{i=1}^{N_{\text {wall }}} v_{x i}(0) v_{x i}\left(t_{0}\right) .
\end{aligned}
$$

At time $t_{0}$, the part of molecules that will collide with the solid walls in the succeeding time interval equals $C^{D}\left(t_{0}\right)$. Substituting eq. (9) into eq. (12), we have

$$
\Delta C_{\mathrm{wall}}^{D}\left(t_{0}\right)=-\frac{c_{m}}{\sqrt{\pi} L} C^{D}\left(t_{0}\right) \Delta t .
$$

Substituting eqs. (13) and (7) into eq. (6), after solving the differential equation, we have

$$
C^{D}(t)=C^{D}(0) \exp \left[-\left(1+\phi_{D}\right) \vartheta t\right]
$$

with

$$
\phi_{D}=\frac{c_{m}}{\sqrt{\pi} L \vartheta}=\frac{c_{m}}{\sqrt{\pi} L} \times \frac{m D}{k_{\mathrm{B}} T}=\frac{3}{4} K n,
$$

where $K n=\lambda / L$, and $\lambda=1 /\left(\sqrt{2} n \sigma_{T}\right)$.

Substitution of eq. (14) into eq. (3a) gives rise to the diffusion coefficient between the two parallel solid walls,

$$
D^{*}=\int_{0}^{\infty} C^{D}(t) \mathrm{d} t=\frac{C^{D}(0)}{\left(1+\phi_{D}\right) \vartheta}=\frac{D}{1+\phi_{D}},
$$

or

$$
\frac{D^{*}}{D}=\frac{1}{1+3 K n / 4} \text {. }
$$

Similarly, the viscosity and thermal conductivity coefficients are obtained, with the details given in Appendix.

$$
\begin{aligned}
\frac{\eta^{*}}{\eta} & =\frac{1}{1+5 \alpha_{\eta} K n / 4}, \\
\frac{\kappa^{*}}{\kappa} & =\frac{1}{1+39 \alpha_{\kappa} K n / 16} .
\end{aligned}
$$

As derived in Appendix, the parameters $\alpha_{\eta}$ and $\alpha_{\kappa}$ satisfy the following equations, respectively:

$$
\begin{gathered}
\frac{4}{5 \alpha_{\eta}}+\exp \left[-\left(\frac{4}{5}+K n \alpha_{\eta}\right)\right]=1-K n, \\
\frac{8}{15 \alpha_{\kappa}}+\exp \left[-\left(\frac{8}{15}+\frac{13}{10} K n \alpha_{\kappa}\right)\right]=1-\frac{13}{10} K n .
\end{gathered}
$$

As shown in Figure 1, based upon the exact numerical solutions of eqs. (20) and (21), the relations of $\alpha_{\eta}$ and $\alpha_{\kappa}$ to 


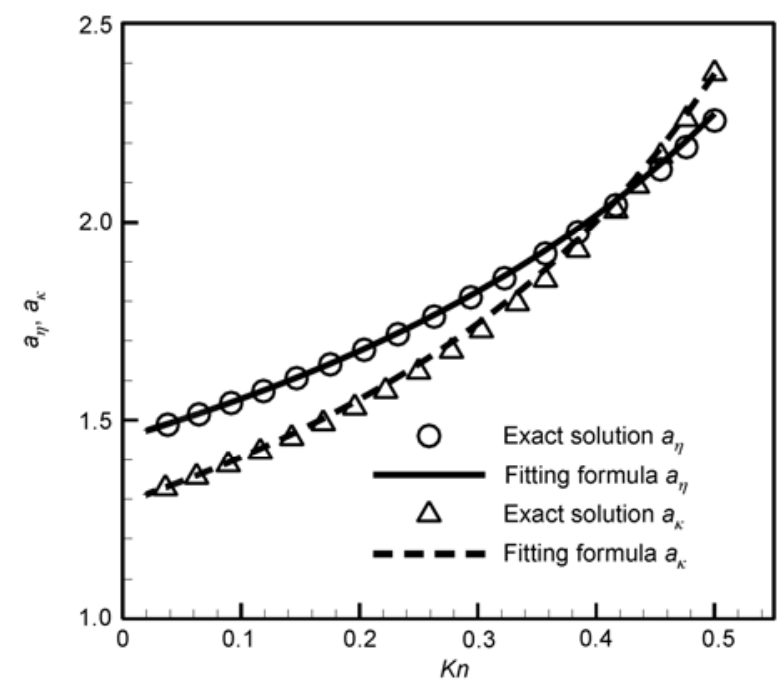

Figure 1 Relations of $\alpha_{\eta}$ and $\alpha_{\kappa}$ to the Knudsen number.

$K n$ can be fitted as:

$$
\begin{gathered}
\alpha_{\eta}=\frac{1}{1-0.9 K n}+0.46, \\
\alpha_{\kappa}=\frac{1}{1-1.04 K n}+0.29 .
\end{gathered}
$$

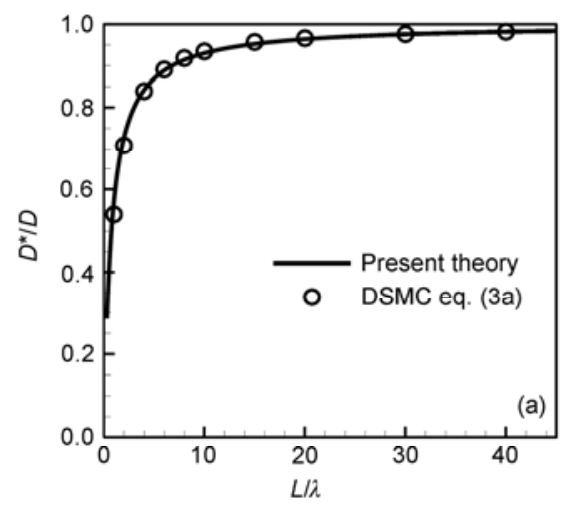

\section{Results and discussion}

The theoretical relations (17), (18) and (19) take into account the effects of the two parallel solid walls on the transport coefficients of gases between them. The first examination employs the case shown in Table 2, which are compared with the present theory in Figure 2. They are in good agreement. They demonstrate that the solid walls result in the nonlinear decrease of transport coefficients as the Knudsen number increases.

Another examination comes from Couette flows and thermal Couette flows. According to eq. (1), the viscosity coefficient is equal to the ratio of the wall shear stress $\tau_{\mathrm{w}}$ to $\Delta U_{\mathrm{w}} / L$ in the Couette flow, and the thermal conductivity coefficient is equal to the ratio of the wall heat flux $q_{\mathrm{w}}$ to $\Delta T_{\mathrm{w}} / L$ in the thermal Couette flow, where $\Delta U_{\mathrm{w}}$ and $\Delta T_{\mathrm{w}}$ are the velocity and temperature differences between the upper and lower plates in these flows, respectively. More computational details on them were described in refs. [10,11], and are not repeated here. As shown in Figures 2(b) and (c), our theories (18) and (19) are in good agreement with the DSMC results based on eq. (1).

Briefly speaking, the physical mechanism for the transport coefficients of gases near a solid wall to decrease lies in the irrelevance of the velocities of a molecules before and

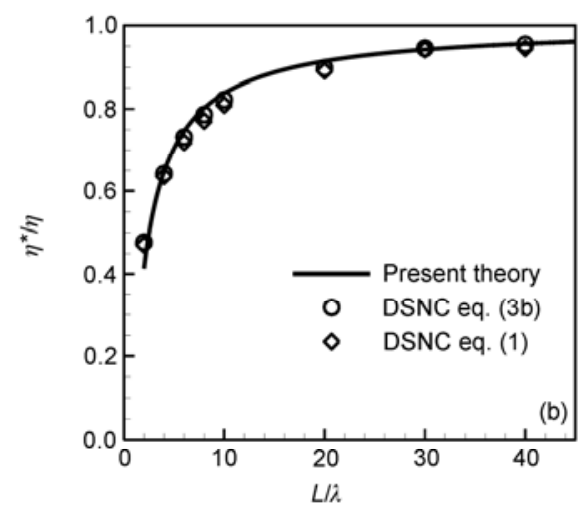

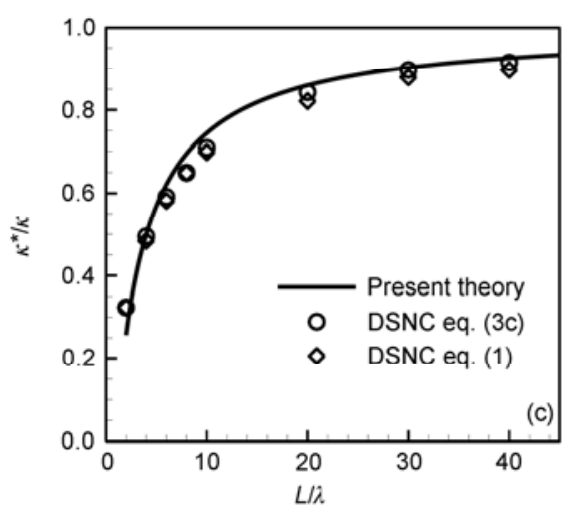

Figure 2 Transport coefficients of argon gas in the standard conditions between two parallel solid walls versus the wall distance. (a) Diffusion; (b) viscosity; (c) thermal conductivity. 
after striking the solid wall. For a typical case of gases between two parallel solid walls, our analysis has established a quantitative relationship between the transport coefficients of gases and the Knudsen number, which is helpful to understanding various transport phenomena of gases around solid walls.

\section{Appendix Viscosity and thermal conductivity coeffi- cients of gases between two parallel solid walls}

The autocorrelation functions of viscosity and thermal conductivity of gases in a system can be written as:

$$
\begin{aligned}
C^{\eta}(t)=\left\langle J^{\eta}{ }_{x y}(0)\right. & \left.J^{\eta}{ }_{x y}(t)\right\rangle=\left\langle\sum_{i=1}^{N} m v_{i x} v_{i y}(0) \sum_{i=1}^{N} m v_{i x} v_{i y}(t)\right\rangle, \\
C^{\kappa}(t) & =\left\langle J^{\kappa}{ }_{y}(0) J^{\kappa}{ }_{y}(t)\right\rangle \\
& =\left\langle\sum_{i=1}^{N}\left[\frac{1}{2} m v_{i}^{2}-\frac{5}{2} k T\right] v_{i y}(0)\right. \\
& \left.\times \sum_{i=1}^{N}\left[\frac{1}{2} m v_{i}^{2}-\frac{5}{2} k T\right] v_{i y}(t)\right\rangle
\end{aligned}
$$

with the initial values

$$
\begin{aligned}
C^{\eta}(0)=\left\langle\sum_{i=1}^{N} m v_{i x} v_{i y}(0) \sum_{i=1}^{N} m v_{i x} v_{i y}(0)\right\rangle=\frac{1}{4} N m^{2} c_{m}^{4}, \\
C^{\kappa}(0)=\left\langle\sum_{i=1}^{N}\left[\frac{1}{2} m v_{i}^{2}-\frac{5}{2} k T\right] v_{i y}(0)\right. \\
\times \sum_{i=1}^{N}\left[\frac{1}{2} m v_{i}^{2}-\frac{5}{2} k T\right] v_{i y}(0) \\
=\frac{5}{16} N m^{2} c_{m}^{6} .
\end{aligned}
$$

Similar to eq. (6), the autocorrelation functions of viscosity and thermal conductivity changed owing to the two different mechanisms, that is

$$
\begin{gathered}
\Delta C^{\eta}\left(t_{0}\right)=C^{\eta}\left(t_{0}+\Delta t\right)-C^{\eta}\left(t_{0}\right)=\Delta C_{\text {bulk }}^{\eta}\left(t_{0}\right)+\Delta C_{\text {wall }}^{\eta}\left(t_{0}\right), \\
\Delta C^{\kappa}\left(t_{0}\right)=C^{\kappa}\left(t_{0}+\Delta t\right)-C^{\kappa}\left(t_{0}\right)=\Delta C_{\text {bulk }}^{\kappa}\left(t_{0}\right)+\Delta C_{\text {wall }}^{\kappa}\left(t_{0}\right) .
\end{gathered}
$$

The first part due to the collisions between molecules themselves decays exponentially [6],

$$
\begin{gathered}
\Delta C_{\text {bulk }}^{\eta}\left(t_{0}\right)=-C^{\eta}\left(t_{0}\right) \xi \Delta t, \\
\Delta C_{\text {bulk }}^{\kappa}\left(t_{0}\right)=-C^{\kappa}\left(t_{0}\right) \chi \Delta t,
\end{gathered}
$$

where $\xi=\frac{\rho c_{m}^{2}}{2 \eta}$ and $\chi=\frac{5 \rho c_{m}^{4}}{8 T \kappa}$.

The second part due to the interaction between the gaseous molecules and solid walls can be treated in analogy to the derivations of eqs. (8)-(12), which gives

$$
\begin{aligned}
\Delta C_{\text {wall }}^{\eta}\left(t_{0}\right)= & -\left\langle\sum_{i=1}^{N} m v_{i x} v_{i y}(0) \sum_{j=1}^{N_{\text {wall }}} m v_{j x} v_{j y}\left(t_{0}\right)\right\rangle, \\
\Delta C_{\text {wall }}^{\kappa}\left(t_{0}\right)= & -\left\langle\sum_{i=1}^{N}\left[\frac{1}{2} m v_{i}^{2}-\frac{5}{2} k T\right] v_{i y}(0)\right. \\
& \left.\times \sum_{j=1}^{N_{\text {wall }}}\left[\frac{1}{2} m v_{j}^{2}-\frac{5}{2} k T\right] v_{j y}\left(t_{0}\right)\right\rangle .
\end{aligned}
$$

Eqs. (a9) and (a10) show that there are two factors to affect the autocorrelation functions of viscosity and thermal conductivity. One is the number of molecules striking the walls $N_{\text {wall }}$, and the other is the velocity component normal to the walls. Assume that $\Delta C_{\text {wall }}^{\eta}\left(t_{0}\right)$ and $\Delta C_{\text {wall }}^{\kappa}\left(t_{0}\right)$ are both proportional to the autocorrelation functions of the molecules colliding with the walls, that is

$$
\begin{gathered}
\frac{\Delta C_{\text {wall }}^{\eta}\left(t_{0}\right)}{C^{\eta}\left(t_{0}\right)}=-\frac{N_{\text {wall }}\left\langle m^{2} v_{i x}{ }^{2} v_{i y}{ }^{2}\right\rangle_{\text {wall }}}{N\left\langle m^{2} v_{i x}{ }^{2} v_{i y}{ }^{2}\right\rangle}, \\
\frac{\Delta C_{\text {wall }}^{\kappa}\left(t_{0}\right)}{C^{\kappa}\left(t_{0}\right)}=-\frac{N_{\text {wall }}\left\langle\left(\frac{1}{2} m v_{i}^{2}-\frac{5}{2} k T\right)^{2} v_{i y}{ }^{2}\right\rangle_{\text {wall }}}{N\left\langle\left(\frac{1}{2} m v_{i}^{2}-\frac{5}{2} k T\right)^{2} v_{i y}{ }^{2}\right\rangle} .
\end{gathered}
$$

It is known that the normal velocity distribution of gaseous molecules colliding with a wall [2,7],

$$
f_{\text {wall }}=\frac{2 v_{y}}{\pi c_{m}{ }^{4}} \exp \left[-\left(v_{x}^{2}+v_{y}{ }^{2}+v_{z}^{2}\right) / c_{m}{ }^{2}\right]
$$

and therefore

$$
\begin{gathered}
\left\langle m^{2} v_{i x}{ }^{2} v_{i y}{ }^{2}\right\rangle_{\text {wall }}=\frac{1}{2} m^{2} c_{m}{ }^{4}, \\
\left\langle m^{2} v_{i x}{ }^{2} v_{i y}{ }^{2}\right\rangle=\frac{1}{4} m^{2} c_{m}^{4}, \\
\left\langle\left(\frac{1}{2} m v_{i}^{2}-\frac{5}{2} k T\right)^{2} v_{i y}{ }^{2}\right\rangle_{\text {wall }}=\frac{13}{16} m^{2} c_{m}{ }^{6}, \\
\left\langle\left(\frac{1}{2} m v_{i}^{2}-\frac{5}{2} k T\right)^{2} v_{i y}{ }^{2}\right\rangle=\frac{5}{16} m^{2} c_{m}{ }^{6} .
\end{gathered}
$$

Substitution of eqs. (a13) and (a14) into eqs. (a11) and (a12) yields

$$
\begin{aligned}
\Delta C_{\text {wall }}^{\eta}\left(t_{0}\right) & =-\frac{2 c_{m} \Delta t}{\sqrt{\pi} L} C^{\eta}\left(t_{0}\right), \\
\Delta C_{\text {wall }}^{\kappa}\left(t_{0}\right) & =-\frac{13 c_{m} \Delta t}{5 \sqrt{\pi} L} C^{\kappa}\left(t_{0}\right) .
\end{aligned}
$$

Contrary to the present analysis that employs a continuous view to treat the autocorrelation, gaseous molecules always collide with a solid wall discretely. To match the 
difference, a modified factor should be introduced:

$$
\begin{gathered}
\Delta C_{\text {wall }}^{\eta}\left(t_{0}\right)=-\alpha_{\eta} \frac{2 c_{m} \Delta t}{\sqrt{\pi} L} C^{\eta}\left(t_{0}\right), \\
\Delta C_{\text {wall }}^{\kappa}\left(t_{0}\right)=-\alpha_{\kappa} \frac{13 c_{m} \Delta t}{5 \sqrt{\pi} L} C^{\kappa}\left(t_{0}\right) .
\end{gathered}
$$

Similar to the derivations of eq. (14), the autocorrelation functions of viscosity are obtained from eqs. (a5), (a7) and (a17), and eqs. (a6), (a8) and (a18), respectively,

$$
\begin{aligned}
& C^{\eta}(t)=C^{\eta}(0) \exp \left[-\left(1+\alpha_{\eta} \phi_{\eta}\right) \xi t\right], \\
& C^{\kappa}(t)=C^{\kappa}(0) \exp \left[-\left(1+\alpha_{\kappa} \phi_{\kappa}\right) \chi t\right],
\end{aligned}
$$

with

$$
\begin{gathered}
\phi_{\eta}=\frac{2 c_{m}}{\sqrt{\pi} L \xi}=\frac{2 c_{m}}{\sqrt{\pi} L} \times \frac{2 \eta}{\rho c_{m}^{2}}=\frac{5}{4} K n, \\
\phi_{\kappa}=\frac{13 c_{m}}{5 \sqrt{\pi} L \chi}=\frac{2 c_{m}}{\sqrt{\pi} L} \times \frac{8 \kappa T}{5 \rho c_{m}^{4}}=\frac{39}{16} K n .
\end{gathered}
$$

Substitution of eq. (a19) and (a20) into the G-K relation (3b) and (3c) yields the viscosity coefficient and thermal conductivity of gases between the parallel solids walls as follows:

$$
\begin{gathered}
\eta^{*}=\frac{1}{k_{\mathrm{B}} V T} \int_{0}^{\infty} C^{\eta}(t) \mathrm{d} t=\frac{1}{k_{\mathrm{B}} V T} \frac{C^{\eta}(0)}{\left(1+\alpha_{\eta} \phi_{\eta}\right) \xi}=\frac{\eta}{1+\alpha_{\eta} \phi_{\eta}}, \\
\kappa^{*}=\frac{1}{k_{\mathrm{B}} V T^{2}} \int_{0}^{\infty} C^{\kappa}(t) \mathrm{d} t \\
=\frac{1}{k_{\mathrm{B}} V T^{2}} \frac{C^{\kappa}(0)}{\left(1+\alpha_{\kappa} \phi_{\kappa}\right) \chi}=\frac{\kappa}{1+\alpha_{\kappa} \phi_{\kappa}}
\end{gathered}
$$

or

$$
\begin{aligned}
\frac{\eta^{*}}{\eta} & =\frac{1}{1+5 \alpha_{\eta} K n / 4}, \\
\frac{\kappa^{*}}{\kappa} & =\frac{1}{1+39 \alpha_{\kappa} K n / 16} .
\end{aligned}
$$

To determine $\alpha_{\eta}$ and $\alpha_{\kappa}$, let us consider a small time interval $\left[t_{0}, t_{0}+\tau_{\mathrm{c}}\right]$, where $\tau_{\mathrm{c}}$ is the mean collision time. For the small interval, approximately,

$$
\begin{aligned}
\Delta C_{\text {wall }}^{\eta}\left(\tau_{\mathrm{c}}\right) & =\int_{t_{0}}^{t_{0}+\tau_{\mathrm{c}}} \Delta C_{\text {wall }}^{\eta}(t)=\int_{t_{0}}^{t_{0}+\tau_{\mathrm{c}}}-\frac{2 c_{m}}{\sqrt{\pi} L} C^{\eta}(t) \mathrm{d} t \\
& \approx \int_{t_{0}}^{t_{0}+\tau_{\mathrm{c}}}-\frac{2 c_{m}}{\sqrt{\pi} L} C^{\eta}\left(t_{0}\right) \mathrm{d} t=-\frac{2 c_{m} \tau_{\mathrm{c}}}{\sqrt{\pi} L} C^{\eta}\left(t_{0}\right) . \text { (a27) }
\end{aligned}
$$

Meanwhile, according to the continuous model (a17), we have

$$
\begin{aligned}
\Delta C_{\text {wall }}^{\eta}\left(\tau_{\mathrm{c}}\right)= & \int_{t_{0}}^{t_{0}+\tau_{\mathrm{c}}} \Delta C_{\text {wall }}^{\eta}(t)=\int_{t_{0}}^{t_{0}+\tau_{\mathrm{c}}}-\frac{2 \alpha_{\eta} c_{m}}{\sqrt{\pi} L} C^{\eta}(t) \mathrm{d} t \\
= & \int_{t_{0}}^{t_{0}+\tau_{\mathrm{c}}}-\frac{2 \alpha_{\eta} c_{m}}{\sqrt{\pi} L} C^{\eta}(0) \exp \left[-\left(1+\alpha_{\eta} \phi_{\eta}\right) \xi t\right] \mathrm{d} t \\
= & -\frac{2 \alpha_{\eta} c_{m}}{\sqrt{\pi} L} \times \frac{C^{\eta}\left(t_{0}\right)}{\left(1+\alpha_{\eta} \phi_{\eta}\right) \xi} \\
& \times\left\{1-\exp \left[-\left(1+\alpha_{\eta} \phi_{\eta}\right) \xi \tau_{\mathrm{c}}\right]\right\} .
\end{aligned}
$$

Let the right side of eq. (a27) equal that of eq. (a28). Then

$$
\frac{4}{5 \alpha_{\eta}}+\exp \left[-\frac{4}{5}\left(1+\alpha_{\eta} \phi_{\eta}\right)\right]=1-\frac{4}{5} \phi_{\eta}
$$

Substituting eq. (a21) into eq. (a29), we have

$$
\frac{4}{5 \alpha_{\eta}}+\exp \left[-\left(\frac{4}{5}+K n \alpha_{\eta}\right)\right]=1-K n \text {. }
$$

The equation to determine $\alpha_{\kappa}$ can be obtained similarly, which is

$$
\frac{8}{15 \alpha_{\kappa}}+\exp \left[-\left(\frac{8}{15}+\frac{13}{10} K n \alpha_{\kappa}\right)\right]=1-\frac{13}{10} K n .
$$

This work was supported by the National Natural Science Foundation of China (Grant No. 10921062).

1 Bird R B, Stewart W E, Lightfoot E N. Transport Phenomena. 2nd ed. New York: John Wiley \& Sons, 2002

2 Chapman S, Cowling T G. The Mathematical Theory of Non-uniform Gases. Cambridge: Cambridge University Press, 1970

3 Einstein A. On the movement of small particles suspended in stationary liquids required by the molecular-kinetic theory of heat. Ann Phys, 1905, 17: 549-560

4 Kubo R, Toba M, Hashitsume N. Statistical Physics II. 2nd ed. Berlin: Springer-Verlag, 1995

5 Helfand E. Transport coefficients from dissipation in a canonical ensemble. Phys Rev, 1960, 119: 1-9

6 McQuarrie D. Statistical Mechanics. New York: Harper \& Row, 2000

7 Bird G A. Molecular Gas Dynamics and the Direct Simulation of Gas Flows. Oxford: Clarendon Press, 1994

8 Petravic J, Harrowell P. Linear response theory for thermal conductivity and viscosity in terms of boundary fluctuations. Phys Rev E, 2005, 71: 061201

9 Zhang J, Fan J, Fei F. Effects of convection and solid wall on the diffusion in microscale convection flow. Phys Fluids, 2010, 22: 122005

10 Fan J, Shen C. Statistical simulation of low-speed rarefied gas flows. J Comput Phys, 2001, 167: 393-412

11 Sun Q H, Boyd I D. A direct simulation method for subsonic, micro-scale flows. J Comput Phys, 2002, 179: 400-425 